\title{
Aberrant GIMAP2 expression affects oral squamous cell carcinoma progression by promoting cell cycle and inhibiting apoptosis
}

\author{
MARI KOMATSU $^{1}$, KENGO SAITO ${ }^{2}$, ISAO MIYAMOTO ${ }^{3}$, KAZUYUKI KOIKE ${ }^{3}$, MANABU IYODA ${ }^{3}$, \\ DAI NAKASHIMA ${ }^{3}$, ATSUSHI KASAMATSU ${ }^{3}$, MASASHI SHIIBA ${ }^{4}$, \\ HIDEKI TANZAWA $^{1,3}$ and KATSUHIRO UZAWA ${ }^{1,3}$
}

\author{
Departments of ${ }^{1}$ Oral Science, ${ }^{2}$ Molecular Virology and ${ }^{3}$ Dentistry and Oral-Maxillofacial Surgery, \\ Chiba University, Chiba 260-8670; ${ }^{4}$ Department of Medical Oncology, \\ Graduate School of Medicine, Chiba University, Chiba 260-8677, Japan
}

Received August 6, 2020; Accepted November 1, 2021

DOI: $10.3892 / \mathrm{ol} .2021 .13167$

\begin{abstract}
GTPases of immunity-associated protein 2 (GIMAP2) is a GTPase family member associated with $\mathrm{T}$ cell survival. However, its mechanisms of action in oral squamous cell carcinoma (OSCC) remain largely unknown. Therefore, the present study aimed to elucidate the possible role of GIMAP2 in OSCC development by investigating its expression levels and molecular mechanisms in OSCC. Reverse transcription quantitative PCR, immunoblotting and immunohistochemistry indicated that GIMAP2 expression was significantly upregulated $(\mathrm{P}<0.05)$ in OSCC-derived cell lines and primary OSCC specimens compared with that in their normal counterparts. GIMAP2-knockdown OSCC cells exhibited decreased cell growth, which was associated with cyclin-dependent kinase (CDK)4, CDK6 and phosphorylated $\mathrm{Rb}$ downregulation and p53 and p21 upregulation. In addition to cell cycle arrest, GIMAP2 affected anti-apoptotic functions in GIMAP2-knockdown cells by upregulating Bcl-2 and downregulating Bax and Bak. These findings indicated that GIMAP2 may significantly influence OSCC development
\end{abstract}

Correspondence to: Dr Kengo Saito, Department of Molecular Virology, Chiba University, 1-8-1 Inohana, Chuo-ku, Chiba 260-8670, Japan

E-mail: saitok@chiba-u.jp

Dr Katsuhiro Uzawa, Department of Oral Science, Chiba University, 1-8-1 Inohana, Chuo-ku, Chiba 260-8670, Japan

E-mail: uzawak@faculty.chiba-u.jp

Abbreviations: GIMAP2, GTPases of immunity-associated proteins 2; OSCC, oral squamous cell carcinoma; HNOK, human normal oral keratinocyte; RT-qPCR, reverse transcription quantitative PCR

Key words: GTPases of immunity-associated proteins 2, oral squamous cell carcinoma, tumor progression, $\mathrm{G}_{1}$ phase cell cycle arrest, p53, anti-apoptosis and apoptosis inhibition and thus is a potential biomarker of OSCC.

\section{Introduction}

Oral cancer, the sixth most common cancer, is a severe and growing problem with an estimated incidence of $\sim 275,000$ cases annually (1). Oral squamous cell carcinoma (OSCC), the commonest type of oral cancer, can occur via numerous processes during which multiple genetic events alter the normal functioning of oncogenes and tumor suppressor genes. Cancer-related genes display the following six fundamental features: Growth signal self-sufficiency, insensitivity to growth-inhibitory signals, apoptosis evasion, limitless replicative potential, sustained angiogenesis and the ability to invade and metastasize (2). Previous studies have shown that OSCC development is associated with cell proliferation and apoptosis rates (3-5); thus, it is necessary to understand the mechanisms underlying malignant tumors to develop new and effective treatment strategies.

GTPases of immunity-associated proteins (GIMAPs), also known as immunity-associated nucleotide binding proteins or IMAPs, are a family of GTPases found in vertebrates and plants. Humans have seven GIMAPs clustered on chromosome 7, consisting of an amino-terminal guanine-nucleotide binding domain (G-domain) followed by varying C-terminal extensions of 50-100 amino acids long (6,7). GIMAPs have seven isoforms expressed in humans (namely, GIMAP1, GIMAP2, GIMAP4-GIMAP8; GIMAP3 is a pseudogene) (8), regulating $\mathrm{T}$ cell survival during their development, selection and homeostasis and they may be linked to the onset of T lymphopenia, leukemia and autoimmunity $(9,10)$. GIMAPs may also be involved in the mitochondrial regulation of lymphocyte apoptosis by interacting with the Bcl-2 family proteins (8). In addition, it has been suggested that GIMAP3 and GIMAP5 are involved in the mitochondria-mediated apoptosis regulatory pathway (8). GIMAP3 and GIMAP5 have similar primary structures and they are both localized in the intracellular membrane fraction, where several Bcl-2 family 
proteins are located. Furthermore, both GIMAP3 and GIMAP5 co-immunoprecipitate with Bcl-2 and Bcl-xL in T cells (8). Patterson et al (11) indicate that GIMAP5 is associated with lymphocyte survival, autoimmunity and colitis and is essential for the inactivation of glycogen synthase kinase- $3 \beta$ following $\mathrm{T}$ cell activation. The preliminary examination of the mRNA expression levels of GIMAP1, GIMAP2, GIMAP4-GIMAP8 in OSCC the present study revealed only GIMAP2 expression to be significantly $(\mathrm{P}<0.05$; Fig. $1 \mathrm{~A}$ and $\mathrm{B})$ increased compared with that in HNOKs with the other isoforms showing low expression and GIMAP4 and GIMAP7 not being expressed (Fig. S1).

GIMAP2, expressed in humans with no orthologs in mice and rats, is the second-largest protein in the human GIMAP family containing two C-terminal hydrophobic regions (12). According to BioGPS (https://www.biogps.org), T cells, blood cells, including platelets and the spleen express GIMAP2. To the best of the authors' knowledge, there are only two studies on GIMAP2. In one of the studies, Schwefel et al (6) showed that GIMAP2 assembles into a GTP-dependent scaffold and the C-terminal amino acid stretch targets GIMAP2 toward lipid droplets. In the other study, Schwefel et al (7) found that GIMAP2 expression was maintained in all the examined human lymphoma $\mathrm{T}$ cell lines, whereas the expression of other GIMAP members was inhibited in these tumor cell lines. This is in line with the observations of the present study and suggests a favorable role of GIMAP2 in cancer cell survival. Certain GIMAPs may be associated with $\mathrm{T}$ lymphopenia and leukemia $(9-11,13)$, although the biological functions of most GIMAPs, including GIMAP2, remain to be elucidated. As the primary function of GIMAP2 in the progression of solid cancers, such as OSCC, is currently unclear, remain investigated its expression and molecular mechanisms in OSCC.

\section{Materials and methods}

Cell and tissue samples. Human OSCC-derived cell lines, including HSC-2 (RBRC-RCB1945; oral cavity), HSC-3 (JCRB-0623; tongue), HSC-4 (RBRC-RCB1902, tongue), HSC3-M3 (JCRB-1354, tongue), Sa3 (RBRC-RCB0980, gingiva), Ho-1-N-1 (JCRB-0831, buccal mucosa), KOSC2 (JCRB-0126.1, mouth floor), SAS (RBRC-RCB 1974, tongue) and Ho-1-u-1 (RBRC-RCB2102, mouth floor), were purchased from the JCRB Cell Bank and RIKEN BioResource Center. All cancer cells were cultured in low-glucose Dulbecco's modified Eagle's medium (DMEM; Sigma-Aldrich; Merck KGaA) supplemented with $10 \%$ heat-inactivated fetal bovine serum (FBS; HyClone; Cytiva) and $50 \mathrm{U} / \mathrm{ml}$ of penicillin and streptomycin at $37^{\circ} \mathrm{C}$ in a humidified $5 \%$ carbon dioxide atmosphere. Human normal oral keratinocytes (HNOKs) were obtained from three healthy donors. The donors comprised 2 men and 1 woman. The donors were 27, 28 and 22 years old, respectively, and were recruited between April 2017 and June 2017. HNOKs were cultured in Oral Keratinocyte Medium New Zealand BPE (ScienCell Research Laboratories, Inc.; cat. no. 2611) as described previously $(14,15)$. The ethics committee of the Graduate School of Chiba University approved this study (protocol number 680). All patients provided written informed consent prior to their inclusion in the study.
mRNA expression analysis. The present study performed reverse transcription quantitative PCR (RT-qPCR) (14-18) .Cell were grown to $80 \%$ confluence in a $10-\mathrm{cm}$ dish. Total RNA was isolated using TRIzol ${ }^{\circledR}$ Reagent (Invitrogen; Thermo Fisher Scientific, Inc.; cat. no. 15596018), according to the manufacturer's instructions. cDNA was generated using ReverTra Ace qPCR RT Master Mix (Toyobo Life Science; cat. no. FSQ-201) according to the manufacturers' instructions. RT-qPCR was performed in a $20-\mu 1$ reaction volume using FastStart SYBR-Green Master (Roche Diagnostics; cat. no. 4673492001) according to the manufacturer's protocol $(17,18)$. The following primers were used to amplify GIMAP2: GIMAP2 No. 1, forward, 5'-CGATTCAAATGC TTGCTTCC-3' and reverse, 5'-GGACCAAAATGAACA CAGTCAC-3' (Thermo Fisher Scientific, Inc., Waltham, MA, USA); GIMAP2 No. 2, forward, 5'-TGGAAGGACCACTGT GAAGC-3' and reverse, 5'-GTCCTGTGAGGTATAGCG GC-3' (Greiner Bio-One Co Ltd.); and GIMAP2 No. 3 forward, 5'-GGATGCCATGGGACACACAA-3' and reverse, 5'-TAA AGGCACAGATTCGCCCA-3' (Greiner Bio-One Co Ltd.).

In addition, the following primers and universal probes were used: GIMAP1, forward, 5'-TCGAGCTCCTCTCTGGTT ATG-3' and reverse, 5'-TGCAGTCTCAGCCTATGCAC-3' (Thermo Fisher Scientific, Inc.); GIMAP4, forward, 5'-ACA CCAGGGGCCAGTTATG-3' and reverse, 5'-TGCTGTTTC CTGTTGCACTT-3' (Thermo Fischer Scientific); GIMAP5, forward, 5'-TGGGGGACACACTCCATAAT-3' and reverse, 5'GCAGACGCAGTTAAGGAGGA-3' (Thermo Fisher Scientific, Inc.); GIMAP6, forward, 5'-GATGGAGGAAGAAGAATA TGAACAA-3' and reverse, 5'-TTCTGTTCTTTCTCCCTT AGACCT-3' (Thermo Fisher Scientific, Inc.); GIMAP7, forward, 5'-CTCTAGAACTTAGGCACGTACAAGAC-3' and reverse 5'-CTCTAGAACTTAGGCACGTACAAGAC-3' (Thermo Fisher Scientific, Inc.); GIMAP8, forward, 5'-CAGATATAG TGCCTTCAACTACCG-3' and reverse, 5'-GGACAATGT TCAGGGTTTCTTT-3' (Thermo Fisher Scientific, Inc.); and glyceraldehyde 3-phosphate dehydrogenase (GAPDH), forward, 5'-AGCCACATCGCTCAGACA-3' and reverse, 5'-GCCCAA TACGACCAAATCC-3' (Thermo Fisher Scientific, Inc.). The transcript amounts for the target genes were estimated from the respective standard curves and normalized to the GAPDH.

A LightCycler 480 PCR system (Roche Diagnostics) was used with the following RT-qPCR conditions: initial denaturation at $95^{\circ} \mathrm{C}$ for $10 \mathrm{~min}, 45$ amplification cycles of denaturation at $95^{\circ} \mathrm{C}$ for $10 \mathrm{sec}$, annealing at $60^{\circ} \mathrm{C}$ for $30 \mathrm{sec}$ and extension at $72^{\circ} \mathrm{C}$ for $1 \mathrm{sec}$, followed by a cooling step at $40^{\circ} \mathrm{C}$ for $30 \mathrm{sec}$. This experiment was performed in triplicate.

Western blotting. Protein extraction and immunoblotting were conducted as previously described $(16,18,19)$. Cells were washed three times with cold phosphate buffered saline (FUJIFILM Wako Pure Chemical Corporation; cat. no. 045-29795) and gently and briefly centrifuged $\left(11,000 \mathrm{x} \mathrm{g} ; 4^{\circ} \mathrm{C} ; 5 \mathrm{~min}\right)$. The cell pellets were then incubated at $4^{\circ} \mathrm{C}$ for $10 \mathrm{~min}$ in a lysis buffer (7 M urea, $2 \mathrm{M}$ thiourea, 4\% w/v CHAPS, and $10 \mathrm{mM}$ Tris, $\mathrm{pH}$ 7.4). The total protein concentration was measured using a dye-binding method based on the Bradford assay with Bio-Rad Protein Assay Dye Reagent Concentrate (Bio-Rad Laboratories, Inc.; cat. no. 5000006JA). A total of $20 \mu \mathrm{g}$ of the protein was loaded per lane. 
Protein extracts were electrophoresed on $4-12 \%$ Bis-Tris gel (Invitrogen; Thermo Fisher Scientific, Inc.; cat. no. NP0336BOX) and transferred to nitrocellulose membranes (Invitrogen; Thermo Fisher Scientific, Inc.; cat. no. 77010) and blocked for $1 \mathrm{~h}$ at room temperature $\left(25^{\circ} \mathrm{C}\right)$ with Blocking One (Nacalai Tesque, Inc.; cat. no. 03953-95). The membranes were then incubated with polyclonal rabbit anti-GIMAP2 antibody (Rabbit polyclonal antibody specific for human GIMAP2; cat. no. HPA013589; 1:100; Atlas Antibodies). In addition, the following antibodies were used: p21 (Mouse monoclonal antibody specific for human p21; cat. no. sc-6246, 1:200; Santa Cruz Biotechnology, Inc., Inc.), cyclin-dependent kinase (CDK)4 (Mouse monoclonal antibody specific for human CDK4; cat. no. sc-23896, 1:500; Santa Cruz Biotechnology, Inc.), CDK6 (Mouse monoclonal antibody specific for human CDK6; cat. no. sc-7961, 1:200; Santa Cruz Biotechnology, Inc.), Cyclin D1 (Mouse monoclonal antibody specific for human Cyclin D1; cat. no. sc-20044, 1:200; Santa Cruz Biotechnology, Inc.), Cyclin E (Mouse monoclonal antibody specific for human Cyclin E; cat. no. sc-377100, 1:100; Santa Cruz Biotechnology, Inc.), CDK2 (Mouse monoclonal antibody specific for human CDK2; cat. no. sc-6248, 1:200; Santa Cruz Biotechnology, Inc.), p53 (Mouse monoclonal antibody specific for human p53; cat. no. sc-393031, 1:100; Santa Cruz Biotechnology, Inc.), $\mathrm{Rb}$ (Mouse monoclonal antibody specific for human $\mathrm{Rb}$, NBP2-54476IR, 1:200; Novus Biologicals), phosphorylated (p)-Rb (Ser780; Rabbit polyclonal antibody specific for human p-Rb (Ser780); cat. no. ab47763, 1:500; Abcam), Bcl-2 (Mouse monoclonal antibody specific for human Bcl-2; cat. no. sc-7382, 1:50; Santa Cruz Biotechnology, Inc.), Bak (Mouse monoclonal antibody specific for human Bak; cat. no. sc-517390, 1:200; Santa Cruz Biotechnology, Inc.), Bax (Mouse monoclonal antibody specific for human Bax; cat. no. sc-7480, 1:200; Santa Cruz Biotechnology, Inc.), Bcl-xL (Mouse monoclonal antibody specific for human Bcl-xL; cat. no. sc-8392, 1:200; Santa Cruz Biotechnology, Inc.) and mouse $\alpha$-tubulin (Mouse monoclonal antibody specific for human $\alpha$-tubulin; cat. no. sc-5286, 1:1,000; Santa Cruz Biotechnology, Inc.) overnight at $4^{\circ} \mathrm{C}$. The membranes were then washed with TBS-T (1\% Tween) and incubated with horseradish peroxidase-conjugated anti-rabbit IgG (Promega Corporation; cat. no. W4011) or anti-mouse IgG as a secondary antibody (Promega Corporation; cat. no. W4021), for $1 \mathrm{~h}$ at room temperature $\left(25^{\circ} \mathrm{C}\right)$. Finally, the membranes were developed using Clarity Western ECL Substrate (Bio-Rad Laboratories, Inc.; cat. no. 170-5061), and immunoblotting was visualized with the ChemiDoc XRS Plus system (Bio-Rad Laboratories, Inc.). The signal intensities were quantitated using the Image Lab system 6.1 Software (Bio-Rad Laboratories, Inc.). Densitometric GIMAP2 protein data were normalized to $\alpha$-tubulin protein levels.

Immunohistochemistry (IHC) analysis. IHC analysis was performed using 100 tissue samples according to a previously described scoring system $(18,20-24)$. To determine the cut-off value for the GIMAP2 IHC clinical parameter scores, the scores of 100 samples were evaluated by receiver operating characteristic (ROC) curve analysis using a bell curve in Microsoft Excel (Microsoft Corporation) and Excel Statistics (Social Survey Research Information Co., Ltd.). Samples with a score above the cut-off value were defined as GIMAP2-positive.
Polyclonal rabbit anti-GIMAP2 antibody (Rabbit polyclonal antibody specific for human GIMAP2; cat. no. HPA013589, 1:50; Atlas Antibodies) was used as the primary antibody and Dako EnVision+ System- HRP Labeled Polymer Anti-Rabbit (Agilent Technologies, Inc.; cat. no. K4003) was used as the secondary antibody.

Gene expression data for patients with head and neck squamous cell carcinoma (HNSCC) was downloaded from The Cancer Genome Atlas (TCGA) project webpage (http://cancergenome.nih.gov). In total, 499 patients with complete data were selected (i.e., each had a dataset of microRNA expression and publicized clinical information).

Transfection. In a 6-well tissue culture plate, HSC-2 and HSC-3 cells were cultured to a $50-70 \%$ confluency in antibiotic-free DMEM supplemented with FBS. Stable knockdown transfectants were established by transfecting the cell lines (HSC-2 and HSC-3) with GIMAP2-targeting short hairpin (sh)RNA [shGIMAP2; GIMAP2 shRNA Plasmid(h): cat. no. sc-89424-SH; Santa Cruz Biotechnology, Inc.] and control shRNA (shMock) (Control shRNA Plasmid-A: cat. no. sc-108060; Santa Cruz Biotechnology, Inc.) using Lipofectamine ${ }^{\circledR} 3000$ (Invitrogen; Thermo Fisher Scientific, Inc.), according to the manufacturer's instructions. The concentration of the shRNA plasmid was $0.1 \mu \mathrm{g} / \mu \mathrm{l}$. The transfection was carried out at $37^{\circ} \mathrm{C}$ for $48 \mathrm{~h}$. Two to three weeks after transfection, viable colonies were transferred to new dishes. shGIMAP2- and shMock-transfected cells were used for further experiments.

The vector GIMAP2 Human Untagged Clone (OriGene Technologies, Inc.; cat. no. SC101332) was transiently transfected into stable transfectants to confirm the effects of GIMAP2 knockdown. The circular untagged cloning vector PCMV6-XL4 (OriGene Technologies, Inc.; cat. no. PCMV6XL4) was used as the negative control. Stable transfectants were isolated using low-glucose DMEM (Sigma-Aldrich; Merck KGaA) supplemented with $10 \%$ heat-inactivated FBS, $50 \mathrm{U} / \mathrm{ml}$ penicillin and streptomycin and $1 \mu \mathrm{g} / \mathrm{ml}$ puromycin (Santa Cruz Biotechnology, Inc.) at $37^{\circ} \mathrm{C}$ in a humidified $5 \%$ carbon dioxide atmosphere.

Proliferation assay and cell cycle analysis. Proliferation assays were performed as previously described $(16,18,19,25)$. Cell cycle was analyzed by flow cytometry with a BD Accuri C6 Flow Cytometer (Becton-Dickinson and Company) and FlowJo 10.5.3 software (FlowJo LLC) as previously described (21,26-28).

Caspase 3/7 activity assay. GIMAP2-knockdown cells (HSC-2 shGIMAP2 and HSC-3 shGIMAP2) and shMock cells

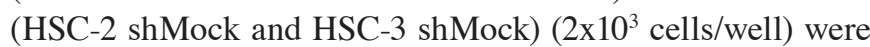
seeded in white-walled 96-well plates and cultured for 2, 4, or 6 days. The activity levels of caspases-3/7 were analyzed using the Caspase-Glo 3/7 assay system, according to the manufacturer's instructions (Promega Corporation; cat. no. G8091). Briefly, the plates were equilibrated to room temperature $\left(25^{\circ} \mathrm{C}\right)$. Caspase-Glo $3 / 7$ reagent $(100 \mu \mathrm{l})$ was added into each well. Following incubation at room temperature for $30 \mathrm{~min}$, the luminescence signal was detected with Synergy HTX (BioTek Instruments, Inc.). 
Statistical analysis. Wilcoxon signed-rank test $(\mathrm{P}<0.05)$ was performed to identify significant associations and ROC curve analysis was used to define a cut-off value to confirm whether samples were GIMAP2-positive or -negative for the classified clinical parameters (Fig. 1E). Wilcoxon signed-rank test was used to compare the median values of paired samples. Furthermore, areas under the curve were determined to confirm the usefulness of this method (Fig. 1G). Dunnett's test was used for the analysis of data shown in Figs. 1A and B, 2A and B, 3A, 4, S1, S2, S3 and S5. Dunnett's post hoc test was performed after the one-way ANOVA. Two-tailed Student's t-test was used for the analysis of data shown in Figs. 1F, 3B and C, 5 and S2.

\section{Results}

GIMAP2 upregulation in OSCC-derived cells. To evaluate GIMAP2 expression, RT-qPCR and western blotting analyses of nine OSCC-derived cell lines and HNOKs was performed. The expression of GIMAP2 mRNA was significantly upregulated $(\mathrm{P}<0.05)$ in two OSCC cell lines compared with that in HNOKs (Fig. 1A and S2). The GIMAP2 level was significantly upregulated $(\mathrm{P}<0.05$, Dunnett's test) in the four OSCC cell lines compared with that in HNOKs (Fig. 1B). Protein level prediction based on the mRNA level is inaccurate because the mRNA and protein levels do not strictly correlate. As one of the reasons is presumably a mutation in the primer-binding site, the mRNA level was verified in Ho-1-u-1 cells by PCR using two additional primer sets targeting different coding regions. As shown in Fig. S2A, GIMAP2 was not expressed in Ho-1-u-1 cells, resulting in a significant difference compared with that in HNOKs.

GIMAP2 expression in primary OSCCs. Representative IHC data for GIMAP2 immunoreactivity in normal oral tissues and OSCC samples (magnification, x400) are shown in Fig. 1C and D; strong cytoplasmic staining for GIMAP2 was detected in OSCC samples, whereas the normal oral tissues showed negative immunoreactivity. The IHC scores of tissue specimens from 100 patients with OSCC were used to investigate the clinical correlations between GIMAP2 expression and pathological characteristics. GIMAP2 expression was significantly higher in OSCC tissues compared with normal oral tissues ( $\mathrm{P}<0.05$; Fig. 1E). The GIMAP2 IHC scores of the adjacent normal tissues ranged from 21.2 to 134.5 (median, 44.6); whereas those of the OSCC tissues ranged from 30.2 to 148.0 (median, 109.8). Gene expression data analysis of patients from TCGA revealed that GIMAP2 expression was significantly higher in HNSCC tissues compared with normal oral tissues $(\mathrm{P}<0.05$; Fig. $1 \mathrm{~F})$. To determine the cut-off value for the GIMAP2 IHC scores, a ROC curve analysis was performed, which yielded an area under the curve of 0.81 and a cut-off value of 104.1 (Fig. 1G). The clinical classifications of GIMAP2-positive OSCC were significantly associated $(\mathrm{P}<0.05)$ with $\mathrm{T}$-primary tumors and the OSCC stage (Table I).

Establishment of GIMAP2-knockdown cells. As GIMAP2 was significantly upregulated in OSCC-derived cells (Figs. 1A and B and S2), its expression in GIMAP2-knockdown cells
(HSC-2 shGIMAP2 and HSC-3 shGIMAP2) was investigated. GIMAP2 mRNA and protein expressions were significantly lower in shGIMAP2 cells compared with shMock cells ( $\mathrm{P}<0.05$; Figs. 2A and B and S3). As other GIMAP isoforms were not highly expressed in the OSCC cell lines examined, the GIMAP2 shRNA used might not have affected other GIMAP isoforms, as it is specific for GIMAP2 (P<0.05; Fig. S1).

Growth of GIMAP2-knockdown cells. To investigate the effect of GIMAP2 knockdown on cell growth, a cell proliferation assay was performed and it was found that cell growth was significantly lower in shGIMAP2 cells (HSC-2 shGIMAP2 and HSC-3 shGIMAP2) compared with shMock cells ( $\mathrm{P}<0.05$; Fig. 3A). For validation, the vector GIMAP2 Human Untagged Clone was transiently transfected into stable transfectants to rescue GIMAP2 expression. The present study confirmed that the expression of GIMAP2 protein increased in GIMAP2-transfected shMock cells (HSC-2 shMock GIMAP2 overexpression and HSC-3 shMock GIMAP2 overexpression), but not in the control shMock cells (HSC-2 shMock control and HSC-3 shMock control; Fig. S4). In addition, GIMAP2 was expressed in GIMAP2-transfected cells (HSC-2 shGIMAP2-1 rescue and HSC-3 shGIMAP2-1 rescue), but not in shGIMAP2 cells (HSC-2 shGIMAP2-1 rescue control and HSC-3 shGIMAP2-1 rescue control; Fig. S4). In addition, cell growth was significantly $(\mathrm{P}<0.05)$ higher in GIMAP2-transfected shGIMAP2 cells (HSC-2 shGIMAP2-1 rescue and HSC-3 shGIMAP2-1 rescue) than in shGIMAP2 cells (HSC-2 shGIMAP2-1 rescue control and HSC-3 shGIMAP2-1 rescue control; Fig. 3B).

Cell cycle analysis of GIMAP2-knockdown cells. Cell cycle analysis showed that the percentage of shGIMAP2 cells in the $G_{1}$ phase was significantly higher than that of shMock cells $(\mathrm{P}<0.05$; Fig. $3 \mathrm{C})$. In addition, $\mathrm{G}_{1}$ phase-related protein expression in shGIMAP2 cells revealed CDK4, CDK6 and phosphorylated (p-)Rb (S780) to be downregulated, whereas p53 and p21 were upregulated $(\mathrm{P}<0.05$; Figs. 4 and S5), indicating that shGIMAP2 suppressed proliferation by arresting the cell cycle in the $G_{1}$ phase.

Apoptosis-related protein expression. The GIMAP family, including GIMAP2, is hypothesized to be associated with apoptosis (12). The evaluation of apoptosis-related protein expression in shGIMAP2 cells showed that Bcl-2 was significantly downregulated, whereas Bak and Bax were upregulated $(\mathrm{P}<0.05$; Figs. 4 and S5), suggesting that GIMAP2 may be associated with apoptosis inhibition.

Caspase 3/7 activity assay. To determine whether the expression of GIMAP2 was associated with the inhibition of apoptosis, the caspase-3/7 activity in shGIMAP2 cells was evaluated. Fig. 5 shows that shGIMAP 2 cells exhibited significant activation of caspase-3/7 compared with that in shMock cells on days 4 and 6 .

\section{Discussion}

To the best of the authors' knowledge, the present study is the first to demonstrate that GIMAP2 is upregulated in HNSCC and is positively associated with TNM classification. The 


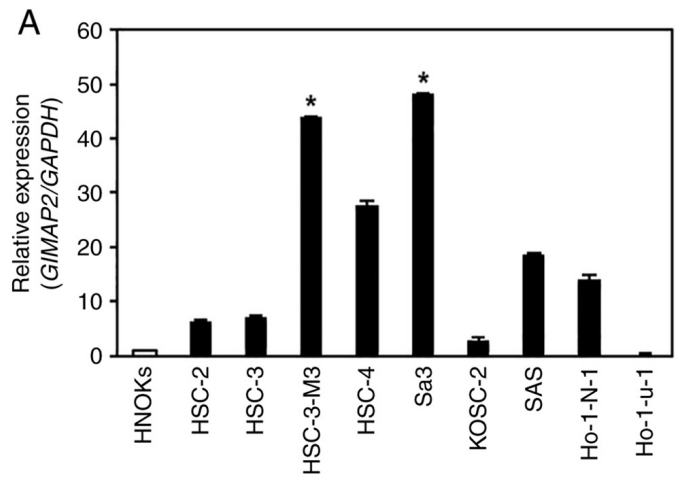

C

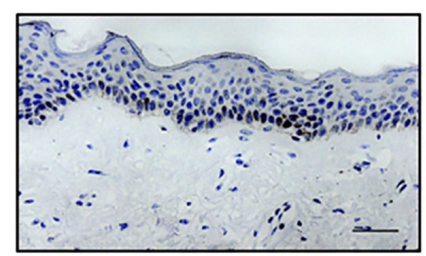

$\mathrm{B}$
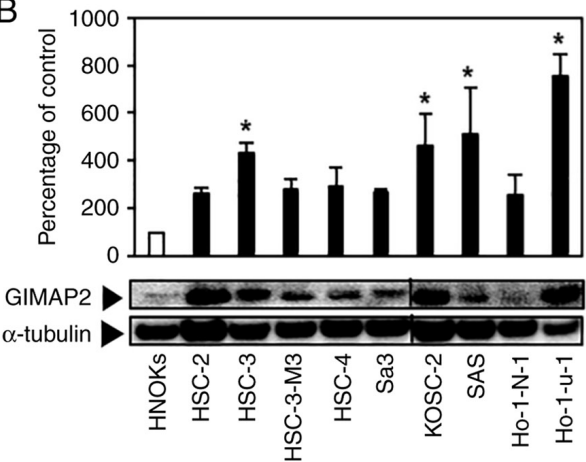

D

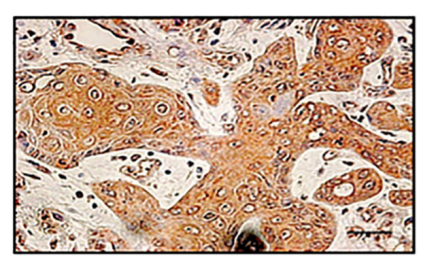

E

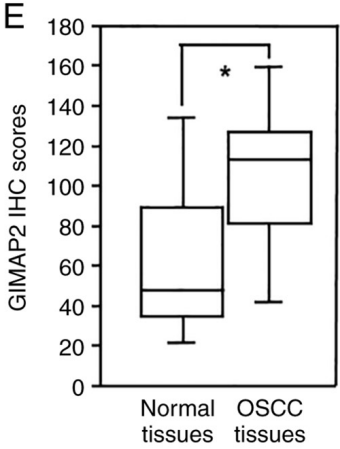

$\mathrm{F}$

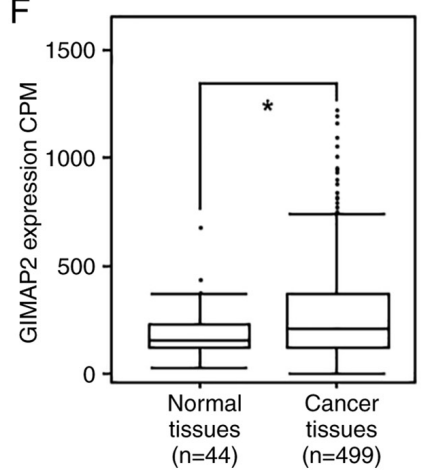

$\mathrm{G}$

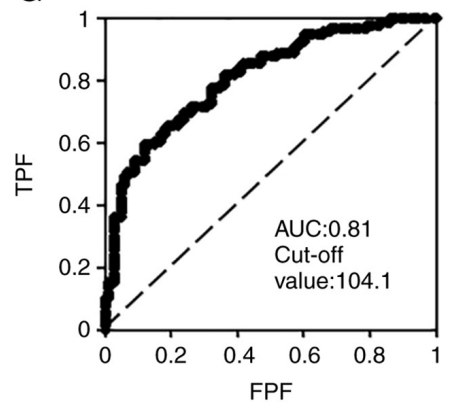

Figure 1. Evaluation of GIMAP2 expression in OSCC-derived cell lines and primary OSCC specimens. (A) Reverse transcription-quantitative PCR analysis showed that GIMAP2 mRNA expression was significantly upregulated (" $\mathrm{P}<0.05$, Dunnett's test) in the two OSCC cell lines compared with that in HNOKs. (B) Western blotting was conducted three times per cell type using GIMAP2 primer No. 1. The results are expressed as mean \pm standard error of the mean of triplicate data. GIMAP2 expression was upregulated ("P<0.05, Dunnett's test) in four OSCC cell lines compared with that in HNOKs. Non-continuous parts of blots probed on the same membrane are indicated using vertical lines. Representative IHC results for GIMAP2 expression in (C) the normal oral tissues (scale bar, $50 \mu \mathrm{m}$ ) and (D) primary OSCC tissues (scale bar, $50 \mu \mathrm{m}$ ). (E) IHC scores showed GIMAP2 expression in primary OSCC (n=100) and normal tissue samples. The GIMAP2 IHC scores of the normal oral tissues and primary OSCC tissues ranged from 21.2 to 134.5 (median, 44.6) and from 30.2 to 148.0 (median, 109.8), respectively. GIMAP2 expression was considerably ("P<0.05, Wilcoxon signed-rank test) higher in OSCC tissues compared in normal oral tissues. (F) The Cancer Genome Atlas data show the GIMAP2 expression status in primary HNSCC; $\mathrm{n}=499$ ) and normal tissue samples (n=44). GIMAP2 expression was considerably higher in HNSCC tissues than in normal tissues ("P<0.05, Student's t-test). (G) The ROC curve analysis indicated that the cut-off value was 104.1 and the AUC was 0.81. GIMAP2, GTPases of immunity-associated proteins 2; OSCC, oral squamous cell carcinoma; HNOK, human normal oral keratinocyte; IHC, immunohistochemistry; ROC, receiver operating characteristic; AUC, area under the curve; TPF, true-positive fraction; FPF, false-positive fraction.
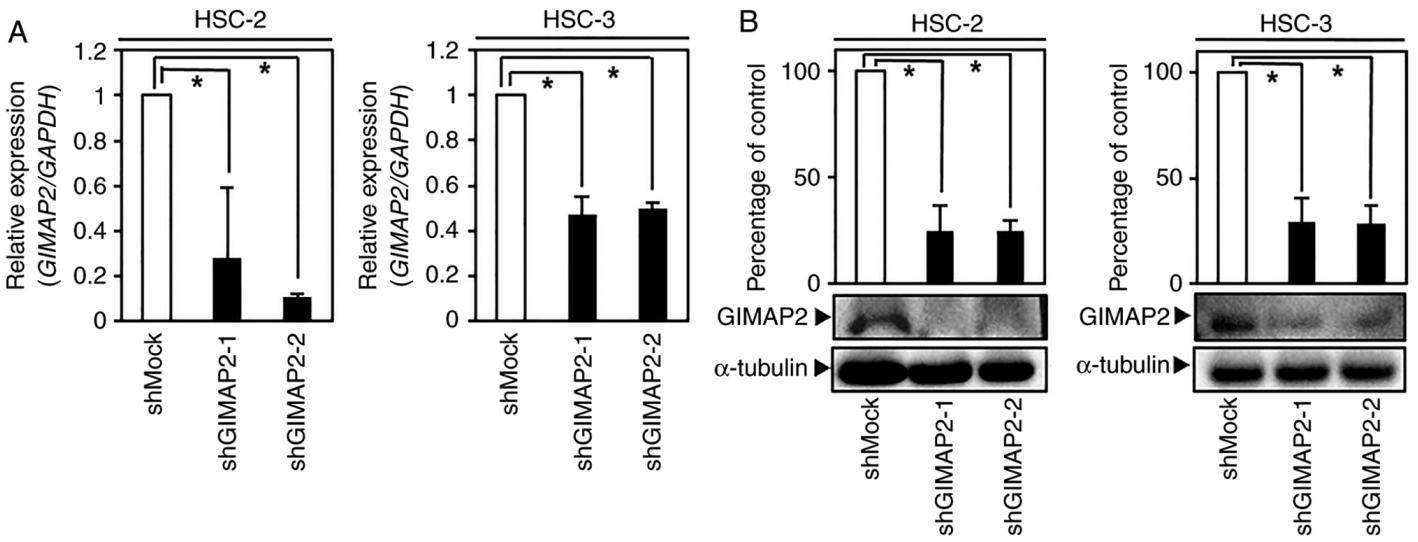

Figure 2. shRNA-mediated GIMAP2 knockdown in OSCC cells (HSC-2 and HSC-3-derived transfectants). (A) GIMAP2 mRNA expression was significantly lower ("P<0.05, Dunnett's test) in shGIMAP2 cells compared with shMock cells. (B) Immunoblotting analysis showed that the GIMAP2 level was lower $($ ( $\mathrm{P}<0.05$, Dunnett's test) in shGIMAP2 cells compared with in shMock cells. Western blotting was conducted three times per cell type. sh, short hairpin; GIMAP2, GTPases of immunity-associated proteins 2; OSCC, oral squamous cell carcinoma. 

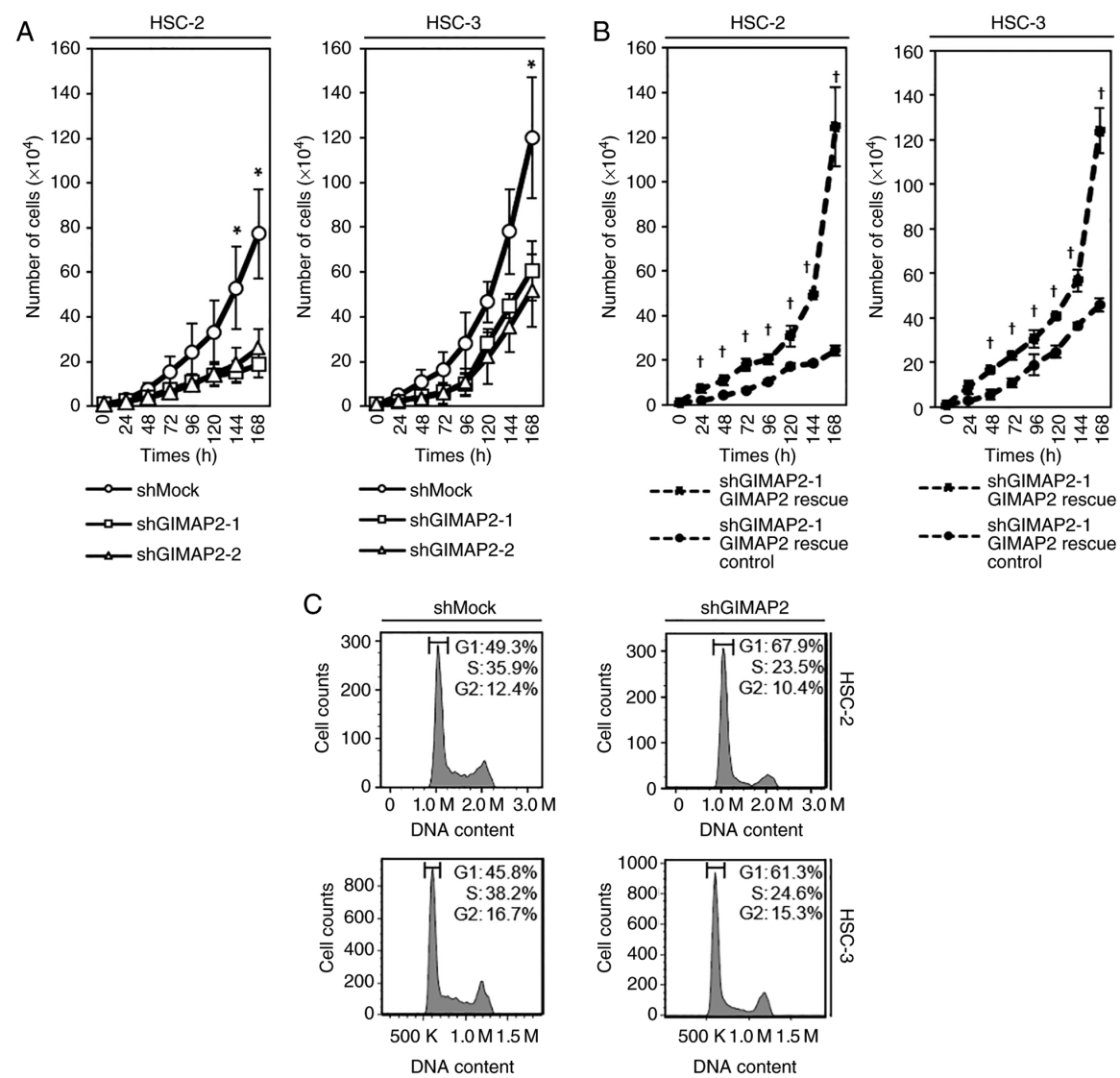

Figure 3. Cell proliferation assay and flow cytometric analysis. (A and B) shGIMAP2 and shMock cells were counted on seven consecutive days. The results are expressed as mean \pm standard error of the mean of triplicate experiments. Cell growth was significantly inhibited ("P $<0.05$, Dunnett's test) in shGIMAP2 cells after $144 \mathrm{~h}$ of culture in HSC-2 cells and after $168 \mathrm{~h}$ of culture in HSC-3 cells. Cell growth was significantly ('P<0.05, Student's t-test) higher in GIMAP2 transiently transfected cells (HSC-2 shGIMAP2-1 rescue and HSC-3 shGIMAP2-1 rescue) than in shGIMAP2 control cells (HSC-2 shGIMAP2-1 rescue control and HSC-3 shGIMAP2-1 rescue control) after $24 \mathrm{~h}$ of culture in HSC-2 cells and after $48 \mathrm{~h}$ of culture in HSC-3 cells. (C) Flow cytometric analysis showed that the percentage of shGIMAP2 cells in the $\mathrm{G}_{1}$ phase was higher compared with shMock cells. sh, short hairpin; GIMAP2, GTPases of immunity-associated proteins 2 .

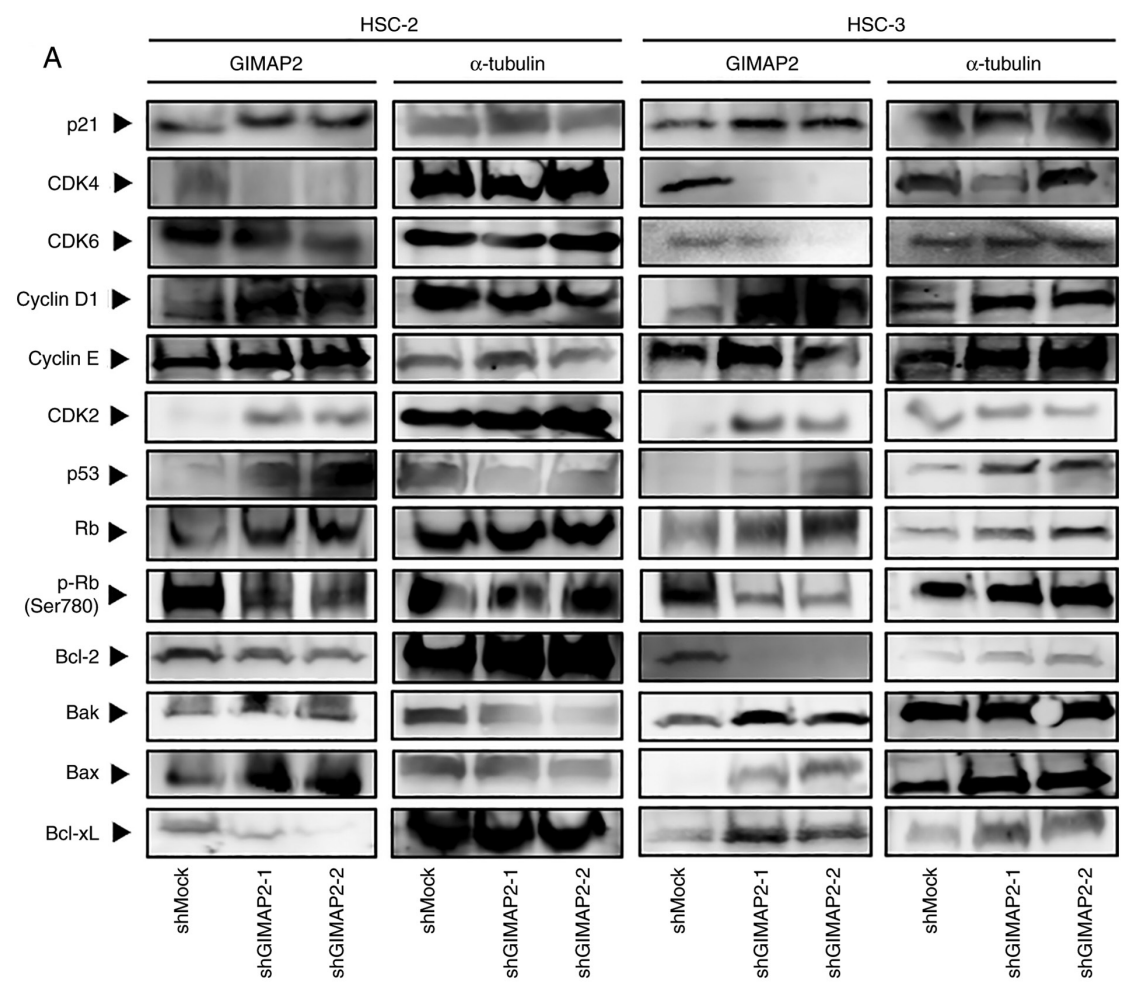

Figure 4. Continued. 
B
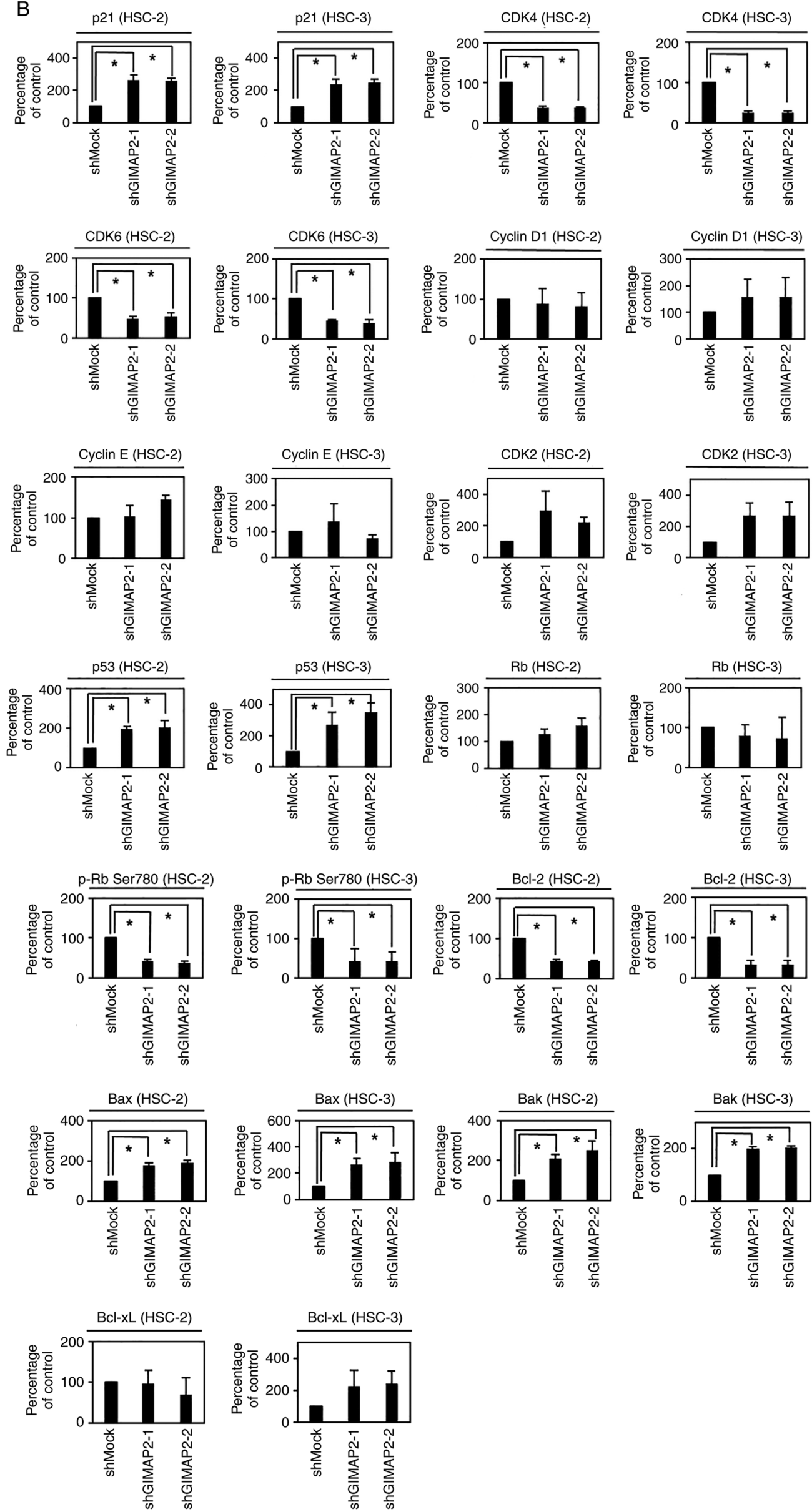

Figure 4. Continued. 

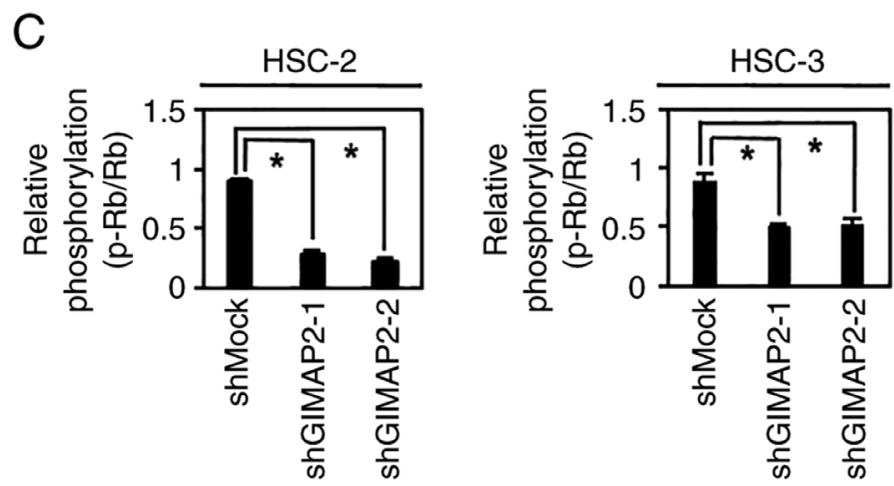

Figure 4. (A, B) Western blotting of proteins associated with cell cycle and apoptosis regulation. Western blotting analyses showed that the p53 and p21 levels were upregulated and CDK4, CDK6 and p-Rb (S780) levels were downregulated in shGIMAP2 cells compared with those in shMock cells ("P<0.05, Dunnett's test). Western blotting analyses showed that the Bak and Bax levels were upregulated and the Bcl-2 level was downregulated in shGIMAP2 cells compared with those in shMock cells ("P<0.05, Dunnett's test). Western blotting was conducted three times per cell type. (C) Western blot analysis revealed the p-Rb protein levels and then normalized to total $\mathrm{Rb}$ ("P<0.05, Dunnett's test). CDK, cyclin-dependent kinase; sh, short hairpin; GIMAP2, GTPases of immunity-associated proteins 2; p-, phosphorylated.

Table I. Correlation between GIMAP2 expression and the clinical classification of oral squamous cell carcinoma.

\begin{tabular}{|c|c|c|c|c|}
\hline \multirow[b]{2}{*}{ Variable } & \multicolumn{4}{|c|}{$\begin{array}{l}\text { Results of immunostaining } \\
\text { No. of patients (\%) }\end{array}$} \\
\hline & Total & GIMAP2 negative & GIMAP2 positive & P-value \\
\hline \multicolumn{5}{|c|}{ Age at surgery (years) } \\
\hline$>70$ & 41 & $14(34)$ & $27(66)$ & $0.580^{\mathrm{a}}$ \\
\hline $60-70$ & 35 & $16(46)$ & $19(54)$ & \\
\hline$<60$ & 24 & $10(42)$ & $14(58)$ & \\
\hline \multicolumn{5}{|l|}{ Sex } \\
\hline Male & 57 & $21(37)$ & $36(63)$ & $0.458^{\mathrm{a}}$ \\
\hline Female & 43 & $19(44)$ & $24(56)$ & \\
\hline \multicolumn{5}{|c|}{ T-primary tumor } \\
\hline $\mathrm{T} 1+\mathrm{T} 2$ & 51 & $32(63)$ & $19(37)$ & $0.0004^{\mathrm{a}, \mathrm{b}}$ \\
\hline $\mathrm{T} 3+\mathrm{T} 4$ & 49 & $8(16)$ & $41(84)$ & \\
\hline \multicolumn{5}{|c|}{ N-regional lymph node } \\
\hline Negative & 63 & $24(38)$ & $39(62)$ & $0.612^{\mathrm{a}}$ \\
\hline Positive & 37 & $16(43)$ & $21(57)$ & \\
\hline \multicolumn{5}{|l|}{ Stage } \\
\hline I & 15 & $10(67)$ & $5(33)$ & $0.004^{\mathrm{a}, \mathrm{b}}$ \\
\hline II & 20 & $12(60)$ & $8(40)$ & \\
\hline III & 18 & $7(39)$ & $11(61)$ & \\
\hline IV & 47 & $11(23)$ & $36(77)$ & \\
\hline \multicolumn{5}{|c|}{ Histopathologic type } \\
\hline Good & 68 & $28(41)$ & $40(59)$ & $0.811^{\mathrm{a}}$ \\
\hline Moderate & 28 & $11(39)$ & $17(61)$ & \\
\hline Poor & 4 & $1(25)$ & $3(75)$ & \\
\hline \multicolumn{5}{|c|}{ Vascular Invasion } \\
\hline Negative & 62 & $24(39)$ & $38(61)$ & $0.737^{\mathrm{a}}$ \\
\hline Positive & 38 & $16(42)$ & $22(58)$ & \\
\hline
\end{tabular}

${ }^{\mathrm{a}} \chi^{2}$ test; ${ }^{\mathrm{b}} \mathrm{P}<0.05$. GIMAP2, GTPase of immunity-associated protein 2 .

knockdown of GIMAP2 revealed that it droves cell proliferation by arresting the cell cycle in the $\mathrm{G}_{1} / \mathrm{S}$ phase and that it may inhibit apoptosis via $\mathrm{Bcl}-2$ upregulation and $\mathrm{Bak}$ and Bax downregulation, suggesting that GIMAP2 serves 


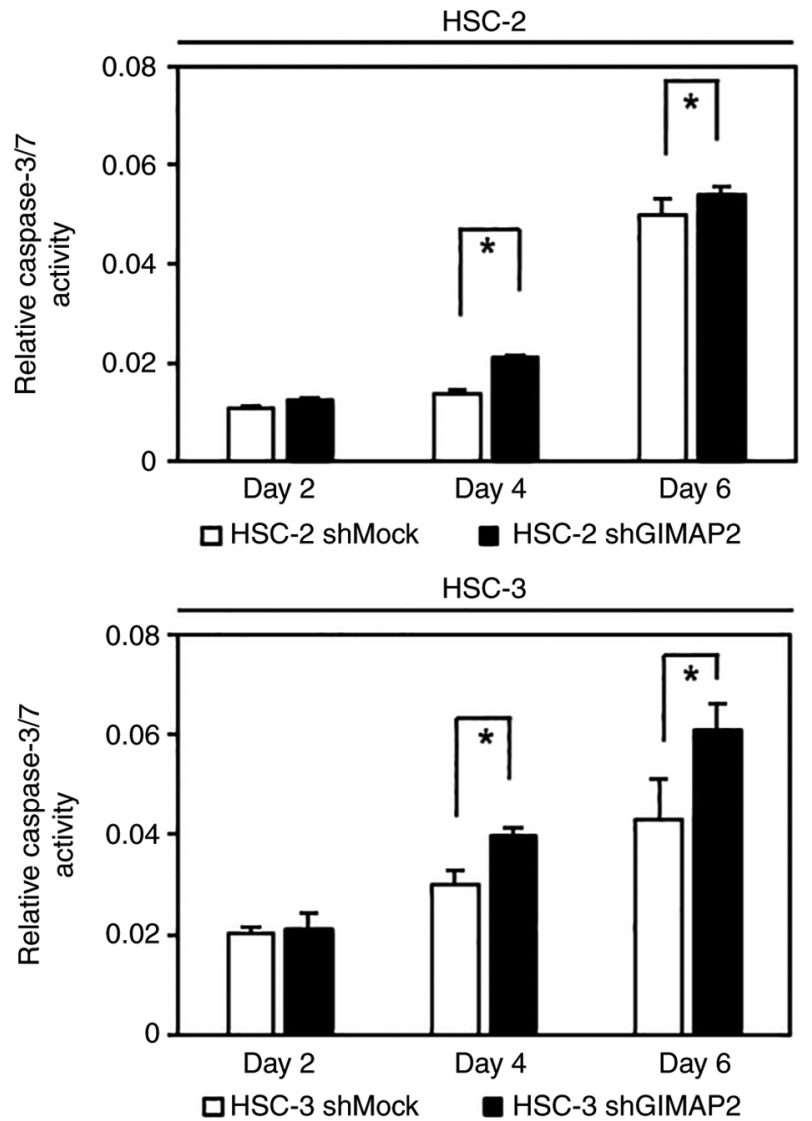

Figure 5. Caspase 3/7 activity. Caspase 3/7 activity in shGIMPA2 HSC-2 and HSC-3 at 2, 4 and 6 days after cell seeding. Data are reported as mean \pm SD $(\mathrm{n}>3)$ relative to the control ("P $<0.05$, Student's t-test). GIMAP2, GTPases of immunity-associated proteins 2 ; sh, short hairpin.

an important role in TNM classification in human OSCC. The cell cycle analysis showed that GIMAP2 knockdown induced $\mathrm{G}_{1} / \mathrm{S}$ phase arrest in OSCC cells and decreased CDK4/CDK6 and p-Rb activities. Recently, the changes in $\mathrm{Rb}$ phosphorylation via CDK4/CDK6 signaling have been reported to control tumor cell proliferation by dysregulating cell cycle progression in several types of tumors, including lung, prostate, head and neck cancers (29-33). Accordingly, the present study confirmed that the expression of GIMAP2 activated the cyclin D1/CDK4/CDK6 complex, which phosphorylates $\mathrm{Rb}$ and results in OSCC proliferation. In addition, increased p53 and p21 expression was observed in GIMAP2-knockdown cells; a study reports that abnormal p53 and p21 signaling correlates with tumor cell proliferation via the $\mathrm{Rb} / \mathrm{E} 2 \mathrm{~F}$ pathway (34), in which E2F activation suppresses the function of p53 and p21 and induces excessive OSCC proliferation. Thus, the data of the present study suggested that GIMAP2 may be associated with cell cycle progression via the $\mathrm{Rb} / \mathrm{E} 2 \mathrm{~F}$ pathway. In addition to cell cycle arrest, p53 regulates apoptosis via the release of cytochrome $\mathrm{c}$ and the transcriptional regulation of pro-apoptotic genes (35). Thus, GIMAP2-knockdown OSCC cells exhibited decreased cell growth, which was associated with CDK4, CDK6 and p-Rb downregulation and p53 and p21 upregulation. The present study found that not only the pro-apoptotic proteins Bak and Bax, but also caspase-3/7 was upregulated in GIMAP2-knockdown cells, whereas the anti-apoptotic protein Bcl-2 was downregulated, consistent with previous findings for other GIMAP family proteins (35). The Bcl-2 family members are associated with cancer development (36), whereas GIMAP2 may be associated with apoptotic signals (37) and inhibit Bcl-2 family-mediated apoptotic signals to support cancer cell survival and development.

Therefore, GIMAP2 may regulate OSCC tumor growth partly via the inhibition of apoptosis; however, further studies are warranted to elucidate the mechanism underlying GIMAP2 anti-apoptotic effects. GIMAP2 expression in OSCC cells was associated with high CDK4, CDK6 and p-Rb expression and low p53 and p21 expression, demonstrating an essential role of GIMAP2 in growth regulation. In addition, GIMAP2 expression was positively associated with $\mathrm{Bcl} 2$ and inversely associated with Bak and Bax expression, which may be indicative of a secondary function of GIMAP2 in controlling apoptosis. Thus, GIMAP2 expression could be a biomarker of OSCC progression and apoptosis inhibition.

\section{Acknowledgements}

The authors would like to thank Ms. Lynda C. Charters for editing and reviewing this manuscript for English language.

\section{Funding}

No funding was received.

\section{Availability of data and materials}

All data generated or analyzed during this study are included in this published article.

\section{Authors' contributions}

MK and HT conceived and designed the study. DN and AK analyzed and interpreted the patient data. MK, IM, KK and MI performed the histological experiments and IHC scoring. $\mathrm{MK}, \mathrm{KS}, \mathrm{KU}$ and MS performed bioinformatics analysis and experiments and interpreted the data. MK and KS drafted and revised the manuscript. KU and HT confirm the authenticity of all the raw data. All authors provided their opinions on the article and data. All authors have read and approved the final manuscript.

\section{Ethics approval and consent to participate}

The ethics committee of the Graduate School of Chiba University approved this study (protocol number 680). All patients provided written informed consent prior to their inclusion in the study.

\section{Patient consent for publication}

Not applicable.

\section{Competing interests}

The authors declare that they have no competing interests. 


\section{References}

1. Warnakulasuriya S: Global epidemiology of oral and oropharyngeal cancer. Oral Oncol 45: 309-316, 2009.

2. Sarkis SA, Abdullah BH, Abdul Majeed BA and Talabani NG: Immunohistochemical expression of epidermal growth factor receptor (EGFR) in oral squamous cell carcinoma in relation to proliferation, apoptosis, angiogenesis and lymphangiogenesis. Head Neck Oncol 2: 13, 2010.

3. Yu T, Liu K, Wu Y, Fan J, Chen J, Li C, Yang Q and Wang Z: MicroRNA-9 inhibits the proliferation of oral squamous cell carcinoma cells by suppressing expression of CXCR4 via the Wnt/ $\beta$-catenin signaling pathway. Oncogene 33: 5017-5027, 2013

4. Hu J, He Y, Yan M, Zhu C, Ye W, Zhu H, Chen W, Zhang C and Zhang Z: Dose dependent activation of retinoic acid-inducible gene-I promotes both proliferation and apoptosis signals in human head and neck squamous cell carcinoma. PLoS One 8: e58273, 2013.

5. Uchida F, Uzawa K, Kasamatsu A, Takatori H, Sakamoto Y, Ogawara K, Shiiba M, Tanzawa H and Bukawa H: Overexpression of cell cycle regulator CDCA3 promotes oral cancer progression by enhancing cell proliferation with prevention of $\mathrm{G}_{1}$ phase arrest. BMC Cancer 12: 321, 2012.

6. Schwefel D, Fröhlich C, Eichhorst J, Wiesner B, Behlke J, Aravind L and Daumke O: Structural basis of oligomerization in septin-like GTPase of immunity-associated protein 2 (GIMAP2). Proc Natl Acad Sci USA 107: 20299-20304, 2010.

7. Schwefel D, Arasu BS, Marino SF, Lamprecht B, Köchert K, Rosenbaum E, Eichhorst J, Wiesner B, Behlke J, Rocks O, et al: Structural insights into the mechanism of GTPase activation in the GIMAP family. Structure 21: 550-559, 2013.

8. Nitta $\mathrm{T}$ and Takahama Y: The lymphocyte guard-IANs: Regulation of lymphocyte survival by IAN/GIMAP family proteins. Trends Immunol 28: 58-65, 2007.

9. Liau WS, Tan SH, Ngoc PC, Wang CQ, Tergaonkar V, Feng H, Gong Z, Osato M, Look AT and Sanda T: Aberrant activation of the GIMAP enhancer by oncogenic transcription factors in T-cel acute lymphoblastic leukemia. Leukemia 31: 1798-1807, 2017.

10. Schnell S, Demolliere C, van den Berk P and Jacobs H: Gimap4 accelerates T-cell death. Blood 108: 591-599, 2006.

11. Patterson AR, Endale M, Lampe K, Aksoylar HI, Flagg A, Woodgett JR, Hildeman D, Jordan MB, Singh H, Kucuk Z, et al: Gimap5-dependent inactivation of GSK3 $\beta$ is required for CD4(+) $\mathrm{T}$ cell homeostasis and prevention of immune pathology. Nat Commun 9: 430, 2018.

12. Nitta T, Nasreen M, Seike T, Goji A, Ohigashi I, Miyazaki T, Ohta T, Kanno M and Takahama Y: IAN family critically regulates survival and development of T lymphocytes. PLoS Biol 4 e103, 2006

13. Patterson AR, Bolcas P, Lampe K, Cantrell R, Ruff B Lewkowich I, Hogan SP, Janssen EM, Bleesing J, Hershey GK and Hoebe K: Loss of GTPase of immunity-associated protein 5 (Gimap5) promotes pathogenic CD4(+) T-cell development and allergic airway disease. J Allergy Clin Immunol 143: 245-257. e6, 2019.

14. Kasamatsu A, Uzawa K, Nakashima D, Koike H, Shiiba M, Bukawa H, Yokoe H and Tanzawa H: Galectin-9 as a regulator of cellular adhesion in human oral squamous cell carcinoma cell lines. Int J Mol Med 16: 269-273, 2005.

15. Endo Y, Uzawa K, Mochida Y, Shiiba M, Bukawa H, Yokoe H and Tanzawa H: Sarcoendoplasmic reticulum $\mathrm{Ca}(2+)$ ATPase type 2 downregulated in human oral squamous cell carcinoma. Int J Cancer 110: 225-231, 2004.

16. Shiiba M, Ishige S, Saito Y, Shimizu T, Minakawa Y,Kasamatsu A, Ogawara K, Uzawa K and Tanzawa H: Down-regulated expression of family with sequence similarity 3 , member B (FAM3B), in oral squamous cell carcinoma. Int J Oral Sci Int 9: 9-16, 2012.

17. Shida-Sakazume T, Endo-Sakamoto Y, Unozawa M, Fukumoto C, Shimada K, Kasamatsu A, Ogawara K, Yokoe H, Shiiba M, Tanzawa $\mathrm{H}$ and Uzawa K: Lysophosphatidylcholine acyltransferase1 overexpression promotes oral squamous cell carcinoma progression via enhanced biosynthesis of platelet-activating factor. PLoS One 10: e0120143, 2015.

18. Saito T, Kasamatsu A, Ogawara K, Miyamoto I, Saito K, Iyoda M, Suzuki T, Endo-Sakamoto Y, Shiiba M, Tanzawa H and Uzawa K: Semaphorin7A promotion of tumoral growth and metastasis in human oral cancer by regulation of $\mathrm{G}_{1}$ cell cycle and matrix metalloproteases: Possible contribution to tumoral angiogenesis. PLoS One 10: e0137923, 2015.
19. Takahara T, Kasamatsu A, Yamatoji M, Iyoda M, Kasama H, Saito T, Takeuchi S, Endo-Sakamoto Y, Shiiba M, Tanzawa H and Uzawa K: SIPA1 promotes invasion and migration in human oral squamous cell carcinoma by ITGB1 and MMP7. Exp Cell Res 352: 357-363, 2017.

20. Koide N, Kasamatsu A, Endo-Sakamoto Y, Ishida S, Shimizu T, Kimura Y, Miyamoto I, Yoshimura S, Shiiba M, Tanzawa $H$ and Uzawa K: Evidence for critical role of lymphocyte cytosolic protein 1 in oral cancer. Sci Rep 7: 43379, 2017.

21. Minakawa Y, Kasamatsu A, Koike H, Higo M, Nakashima D, Kouzu Y, Sakamoto Y, Ogawara K, Shiiba M, Tanzawa H and Uzawa K: Kinesin family member 4A: A potential predictor for progression of human oral cancer. PLoS One 8: e85951, 2013.

22. Baba T, Sakamoto Y, Kasamatsu A, Minakawa Y, Yokota S, Higo M, Yokoe H, Ogawara K, Shiiba M, Tanzawa H and Uzawa K: Persephin: A potential key component in human oral cancer progression through the RET receptor tyrosine kinase-mitogen-activated protein kinase signaling pathway. Mol Carcinog 54: 608-617, 2015.

23. Uzawa K, Kasamatsu A, Saito T, Takahara T, Minakawa Y, Koike K, Yamatoji M, Nakashima D, Higo M, Sakamoto Y, et al: Long-term culture of human odontoma-derived cells with a Rho kinase inhibitor. Exp Cell Res 347: 232-240, 2016.

24. Toeda Y, Kasamatsu A, Koike K, Endo-Sakamoto Y, Fushimi K, Kasama H, Yamano Y, Shiiba M, Tanzawa H and Uzawa K: FBLIM1 enhances oral cancer malignancy via modulation of the epidermal growth factor receptor pathway. Mol Carcinog 57: 1690-1697, 2018

25. Kimura Y, Kasamatsu A, Nakashima D, Yamatoji M, Minakawa Y, Koike K, Fushimi K, Higo M, Endo-Sakamoto Y, Shiiba M, et al: ARNT2 regulates tumoral growth in oral squamous cell carcinoma. J Cancer 7: 702-710, 2016.

26. Miyamoto I, Kasamatsu A, Yamatoji M, Nakashima D, Saito K, Higo M, Endo-Sakamoto Y, Shiiba M, Tanzawa H and Uzawa K: Kinesin family member 14 in human oral cancer: A potential biomarker for tumoral growth. Biochem Biophys Rep 3: 26-31, 2015.

27. Uchida F, Uzawa K, Kasamatsu A, Takatori H, Sakamoto Y, Ogawara K, Shiiba M, Bukawa $\mathrm{H}$ and Tanzawa H: Overexpression of CDCA2 in human squamous cell carcinoma: correlation with prevention of $\mathrm{G}_{1}$ phase arrest and apoptosis. PLoS One 8: e56381, 2013.

28. Hayashi F, Kasamatsu A, Endo-Sakamoto Y, Eizuka K, Hiroshima K, Kita A, Saito T, Koike K, Tanzawa H and Uzawa K: Increased expression of tripartite motif (TRIM) like 2 promotes tumoral growth in human oral cancer. Biochem Biophys Res Commun 508: 1133-1138, 2019.

29. Rader J, Russell MR, Hart LS, Nakazawa MS, Belcastro LT, Martinez D, Li Y, Carpenter EL, Attiyeh EF, Diskin SJ, et al: Dual CDK4/CDK6 inhibition induces cell-cycle arrest and senescence in neuroblastoma. Clin Cancer Res 19: 6173-6182, 2013.

30. Fry DW, Bedford DC, Harvey PH, Fritsch A, Keller PR, Wu Z, Dobrusin E, Leopold WR, Fattaey A and Garrett MD: Cell cycle and biochemical effects of PD 0183812. A potent inhibitor of the cyclin D-dependent kinases CDK4 and CDK6. J Biol Chem 276: 16617-16623, 2001.

31. Patnaik A, Rosen LS, Tolaney SM, Tolcher AW, Goldman JW, Gandhi L, Papadopoulos KP, Beeram M, Rasco DW, Hilton JF, et al: Efficacy and safety of abemaciclib, an inhibitor of CDK4 and CDK6, for patients with breast cancer, non-small cell lung cancer, and other solid tumors. Cancer Discov 6: 740-753, 2016

32. Zi X and Agarwal R: Silibinin decreases prostate-specific antigen with cell growth inhibition via $\mathrm{G}_{1}$ arrest, leading to differentiation of prostate carcinoma cells: Implications for prostate cancer intervention. Proc Natl Acad Sci USA 96: 7490-7495, 1999.

33. Hamilton $\mathrm{E}$ and Infante JR: Targeting CDK4/6 in patients with cancer. Cancer Treat Rev 45: 129-138, 2016.

34. Nevins JR: The Rb/E2F pathway and cancer. Hum Mol Genet 10: 699-703, 2001.

35. Hickman ES, Moroni MC and Helin K: The role of p53 and pRB in apoptosis and cancer. Curr Opin Genet Dev 12: 60-66, 2002.

36. Adams JM and Cory S: The Bcl-2 apoptotic switch in cancer development and therapy. Oncogene 26: 1324-1337, 2007.

37. Schwefel D and Daumke O: GTP-dependent scaffold formation in the GTPase of immunity associated protein family. Small GTPases 2: 27-30, 2011

This work is licensed under a Creative Commons Attribution-NonCommercial-NoDerivatives 4.0 International (CC BY-NC-ND 4.0) License. 